\title{
Cerebrovascular function is preserved during mild hyperthermia in cervical spinal cord injury
}

\author{
Geoff B. Coombs $\mathbb{D}^{1} \cdot$ Diana Vucina $^{2} \cdot$ Hannah G. Caldwell ${ }^{1}$. Otto F. Barak ${ }^{3,4} \cdot$ Tanja Mijacika $^{5} \cdot$ Amanda H. X. Lee $\mathbb{D}^{6} \cdot$ \\ Zoe K. Sarafis $\mathbb{1}^{6} \cdot$ Jordan W. Squair ${ }^{6} \cdot$ Andrei V. Krassioukov $^{6} \cdot$ Aaron A. Phillips $^{7} \cdot$ Zeljko Dujic $^{5} \cdot$ Philip N. Ainslie $^{1}$
}

Received: 31 March 2019 / Revised: 24 June 2019 / Accepted: 25 June 2019 / Published online: 9 July 2019

(c) The Author(s), under exclusive licence to International Spinal Cord Society 2019

\begin{abstract}
Study design Experimental study.

Objectives Compromised cerebrovascular function likely contributes to elevated neurological risk in spinal cord injury (SCI). Passive heating offers many cardiovascular and neurological health benefits; therefore, we aimed to determine the effects of an acute bout of heating on cerebrovascular function in chronic SCI.

Methods Persons with cervical SCI $(n=15)$ and uninjured controls (CON; $n=15)$ completed 60 min of lower limb hot water immersion $\left(40^{\circ} \mathrm{C}\right)$. Assessments of middle cerebral (MCA) and posterior cerebral artery (PCA) velocities, pulsatilities, and neurovascular coupling (NVC) were performed using transcranial Doppler ultrasound. Duplex ultrasonography was used to index cerebral blood flow via the internal carotid artery (ICA), and carotid-femoral pulse-wave velocity (PWV) was measured using tonometry. The NVC response was quantified as the peak hyperemic value during 30 -s cycles of visual stimulation.

Results Mean arterial pressure changed differentially with heating [mean (standard deviation); SCI: $+6(14) \mathrm{mmHg}$, CON: $-8(12) \mathrm{mmHg} ; P=0.01]$. There were no differences in any intracranial artery measures (all $P>0.05$ ), except for small $(\sim 10 \%)$ increases in MCA conductance in CON after heating vs. SCI (interaction $P=0.006)$. Resting ICA flow was greater in SCI vs. CON $(P=0.03)$ but did not change with heating in either group (interaction $P=0.34)$. There were also no between-group differences in the NVC response ( $\triangle \mathrm{PCA}$ conductance) pre- [SCI: 29(19)\% vs. CON: 30(9)\%] or post-heating [SCI 30(9)\% vs. 25(9)\%; interaction $P=0.22$ ].

Conclusions Mild acute heating does not impair or improve cerebrovascular function in SCI or CON. Thus, further study of the effects of chronic heating interventions are warranted.
\end{abstract}

These authors contributed equally: Geoff B. Coombs, Diana Vucina

$\triangle$ Geoff B. Coombs

geoff.coombs91@gmail.com

1 Centre for Heart, Lung and Vascular Health, Faculty of Health and Social Development, University of British Columbia Okanagan, 1147 Research Rd, Kelowna, BC, Canada

2 Department of Neurology, Clinical Hospital Center Split, Spinciceva 1, Split, Croatia

3 Faculty of Medicine, University of Novi Sad, Hajduk Veljkova 3, 21137 Novi Sad, Serbia

4 Faculty of Sports and Physical Education, University of Novi Sad, Novi Sad, Serbia

\section{Introduction}

Cerebrovascular diseases and the risk of stroke are 3-4 fold higher in individuals living with a spinal cord injury (SCI) compared to their uninjured counterparts [1]. The loss of supraspinal control of autonomic function following SCI

5 Department of Integrative Physiology, University of Split School of Medicine, Soltanska 2, HR-21000 Split, Croatia

6 International Collaboration on Repair Discoveries, Faculty of Medicine, University of British Columbia, 818 West 10th Avenue, Vancouver, Canada

7 Departments of Physiology and Pharmacology, Cardiac Sciences, Clinical Neurosciences, Hotchkiss Brain Institute, Libin Cardiovascular Institute, University of Calgary, 3330 Hospital Drive NW, Calgary, AB, Canada 
results in profound blood pressure lability, thereby leading to uncontrolled periods of cerebral hypoperfusion during orthostatic hypotension and hyperperfusion during bouts of autonomic dysreflexia [2, 3]. Both hypo- and hyperperfusion of the brain are associated with cognitive decline and vascular damage $[4,5]$. Neurovascular coupling (NVC), which matches cerebral blood flow (CBF) to neural activity, is also reportedly impaired in SCI [6] and in some neurological diseases (e.g., stroke, Alzheimer's) [7]. Although low resting blood pressure is likely a primary cause of reduced cerebrovascular function in SCI [6, 8], impaired endothelial function, hyposensitivity to vasoconstriction, and profibrotic remodelling might also be contributing factors [9].

Given the reduced physical activity levels following SCI [10], identifying alternative strategies that can be used as an adjunct therapy to help offset elevated cerebrovascular risk are essential. To the best of our knowledge, no studies of cerebrovascular function during hyperthermia have been published in SCI; yet, many of the risk factors associated with cerebrovascular diseases have reportedly been improved using passive heat therapy in uninjured populations. For example, both acute [11] and chronic heating protocols [12] improve arterial stiffness and endothelial function in healthy young and old participants, as well as in peripheral artery disease [13]. Additionally, passive heating causes an acute cytokine response and increases plasma nitrite in overweight men [14]. This cytokine response also occurs acutely in SCI despite attenuated adrenergic activation; [15] however, the effects of heating on nitric oxide bioavailability in SCI are unknown.

The increases in core temperature from the aforementioned studies ranged from $0.4-1.8^{\circ} \mathrm{C}$. Importantly, Bailey et al [16]. demonstrated that eight weeks of repeated lower body hot water immersion $\left(+0.6^{\circ} \mathrm{C}\right.$ core temperature, $30 \mathrm{~min}$ three times per week) were able to increase resting cerebrovascular conductance of the middle cerebral artery (MCA) and attenuate acute hypoperfusion from hyperthermia-induced hypocapnia. Thus, moderate increases in core temperature in the range of $\sim 0.5-0.6^{\circ} \mathrm{C}$ induced by practical methods of immersing the lower limbs in hot water baths have been reported to benefit systemic and cerebral vascular function. Several neurological benefits have recently been reported from chronic heat exposures. The incidence of dementia and Alzheimer's disease and the risk of stroke was observed to be lower in middle-aged men and women who more frequently participated in sauna bathing $[17,18]$. As such, passive heat stress may be a useful strategy to improve cerebrovascular function, particularly for those with SCI who experience greater barriers to physical activity. However, the cerebrovascular and ventilatory responses to heat stress in SCI are unknown and acute increases in core temperature $>0.5^{\circ} \mathrm{C}$ often result in decreased CBF due to hyperventilation-induced hypocapnia in uninjured individuals [19]. Therefore, the aim of this study was to determine the CBF and NVC responses following an acute bout of lower limb heating in SCI participants, with data from uninjured controls $(\mathrm{CON})$ as a comparator. It was hypothesized that heat stress would decrease CBF and NVC due to hypocapnia from heat stress.

\section{Methods}

Ethical approval of this study was provided by the ethics board at the School of Medicine, University of Split, Croatia. Fifteen individuals ( 5 females) with chronic (i.e., $>2$ years) cervical (C3-C7, ASIA A-C) SCI [mean (standard deviation); age: 42 (12) years; BMI: 22 (3)] and 15 age and sex-matched uninjured control participants [CON; age: 42 (11) years; BMI: 25 (3)] completed this protocol. All participants signed informed consent prior to data collection and all procedures conformed to the Declaration of Helsinki. This study shares participants and some data (e.g., core temperature, heart rate, MAP, and baseline NVC) with other manuscripts [20, 21]; however, the hypotheses and cerebrovascular outcomes related to heat stress are unique to the current study.

The protocol comprised $60 \mathrm{~min}$ of lower limb hot water immersion $\left(40^{\circ} \mathrm{C}\right)$ up to the knees with blankets covering the upper body. Baseline measures were performed with the legs immersed in thermoneutral water $\left(33^{\circ} \mathrm{C}\right)$ immediately prior to beginning heating. At the end of heating, the legs were removed from the water bath and CBF was recorded immediately with concurrent measures of end-tidal partial pressure of carbon dioxide $\left(\mathrm{P}_{\mathrm{ET}} \mathrm{CO}_{2}\right)$. These measurements were followed by NVC and PWV $\sim 5$ and $15 \mathrm{~min}$ after heating, respectively. All post-heating measures were collected while core temperature remained elevated and the entire protocol was performed in the supine position. Core temperature (Tcore) was monitored with a telemetric pill (HQInc, Palmetto, FL, USA), heart rate was continuously monitored using a lead II ECG, $\mathrm{P}_{\mathrm{ET}} \mathrm{CO}_{2}$ was measured with a commercial gas analyzer, and arterial blood pressure was measured using finger photoplethysmography and verified every $15 \mathrm{~min}$ with an automated blood pressure cuff. Venous blood samples were processed using a hematology analyzer (AcT8 Hematology Analyzer, Beckman Coulter, Brea, CA, USA). Primary outcomes of CBF, PWV, and $\mathrm{NVC}$ were measured during supine rest $(>15 \mathrm{~min}$ ) prior to heating and within 10-15 min after the end of heating, with no measures collected during heating to facilitate participant comfort.

Transcranial Doppler ultrasound (Spencer Technologies, Redmond, WA, USA) was used to simultaneously measure 


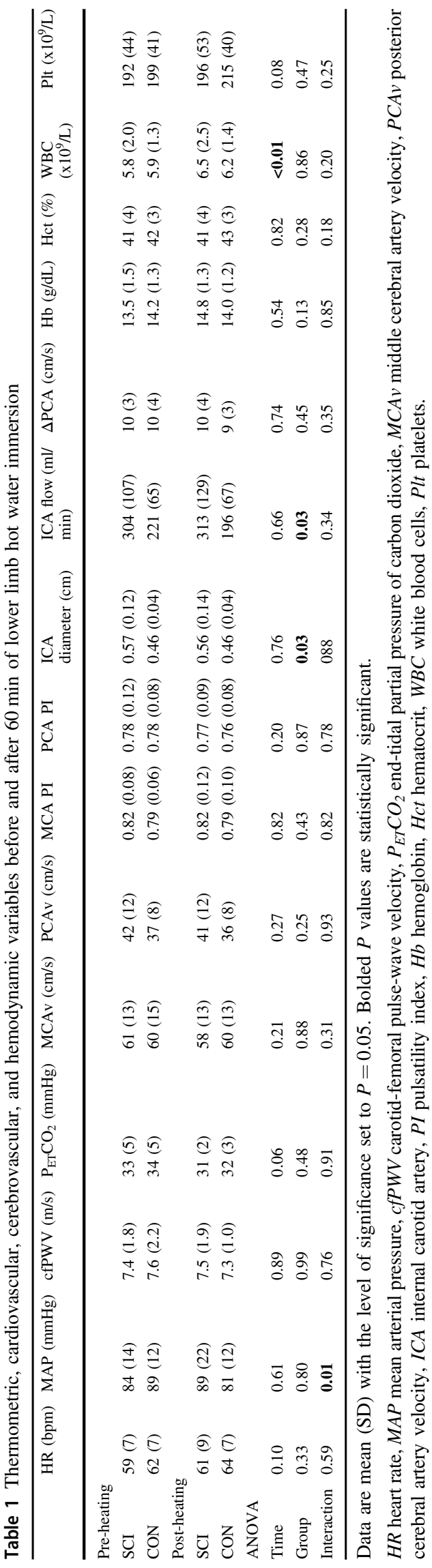

the right middle cerebral artery (MCAv) and the left posterior cerebral artery (PCAv) velocities through acoustic windows located in the temporal region. The NVC response was quantified as the peak hyperemic value in the PCA during 30-s cycles of visual stimulation (i.e., eyes closed/ eyes tracking a moving finger) over a 5-min period to evoke functional changes in cerebral perfusion. Cerebral artery pulsatility index (PI) was estimated in the MCA and PCA (e.g., [systolic MCAv-diastolic MCAv]/mean MCAv). Internal carotid artery (ICA) blood flow was measured using duplex ultrasound to provide an index of CBF. Blood flow was calculated from the 1-min screen-capture recordings as (peak envelope blood velocity/2)* $\left(\pi\left(0.5 *\right.\right.$ diameter $\left.^{2}\right)$ and analyzed using offline automated edge-detection software [22]. Cerebral vascular conductance (CVC) was determined for MCAv, PCAv, and ICA flow by dividing values by mean arterial pressure (MAP). Carotid-femoral PWV (80\% of distance between sites) was measured using a pulse wave tonometer (SPT-301, Millar, Houston, TX, USA) to estimate aortic stiffness, according to international guidelines [23]. Data were analyzed using two-way mixed ANOVAs and are presented as means and standard deviation (SD).

\section{Results}

All cardiovascular and cerebrovascular data are presented in Table 1. Due to inadequate ultrasound image quality (e.g., automatic edge detection software could not properly track vessel walls or MCA/PCA recording did not display typical pulse wave), analyses were not performed on a small number of participants for some measures where indicated. Resting Tcore was $37.10(0.26)^{\circ} \mathrm{C}$ in $\mathrm{CON}$ and 36.37 $(0.62)^{\circ} \mathrm{C}$ in SCI. The increase in Tcore was two-fold greater in SCI compared to the change in $\mathrm{CON}[+0.68(0.17)$ vs. $\left.+0.34(0.17)^{\circ} \mathrm{C} ; \quad P<0.01\right]$. There were no group-byintervention interactions for any of the intracranial measures of MCAv, PCAv, $\mathrm{PCA}_{\mathrm{CVC}}, \mathrm{MCA}_{\mathrm{PI}}$, or $\mathrm{PCA}_{\mathrm{PI}}(n=14$ SCI, $n=13 \mathrm{CON}$; all $P>0.05)$. However, $\mathrm{MCA}_{\mathrm{CVC}}$ decreased by $10 \%$ in SCI and increased by $12 \%$ in CON $(P$ $<0.01$ ), reflecting divergent responses of MAP [SCI: +6 (14) $\mathrm{mmHg}, \mathrm{CON}$ : $-8(12) \mathrm{mmHg} ; P=0.01]$. Although the ICA diameter, flow, and $\mathrm{ICA}_{\mathrm{CVC}}(n=8 \mathrm{SCI}, n=9 \mathrm{CON})$ were all greater in SCI vs. CON $(18 \%, 33 \%$, and $31 \%$, respectively; all $P<0.05)$, there were no group-byintervention interactions (all $P>0.05$ ). There was no group-by-intervention interaction for carotid-femoral PWV $(P=0.76)$, which may be consistent with recent studies of participants with tetraplegia [24]. Peak PCA $_{C V C}$ during visual stimulation also did not present a group-by-time interaction ( $n=13 \mathrm{SCI}, n=10 \mathrm{CON}$; interaction $P=0.22$ ). The pre-heating PCA NVC response was 28.9 (9.0) vs. 29.8 
Fig. 1 Average response of the posterior cerebral artery (PCA) during 30 -s periods of visual stimulation before and after heating. The top panels represent the average relative change in PCAv from a closed eyes baseline and the bottom panels represent the average absolute response. Data are presented as mean (SD) with values corrected for cerebrovascular conductance (CVC)
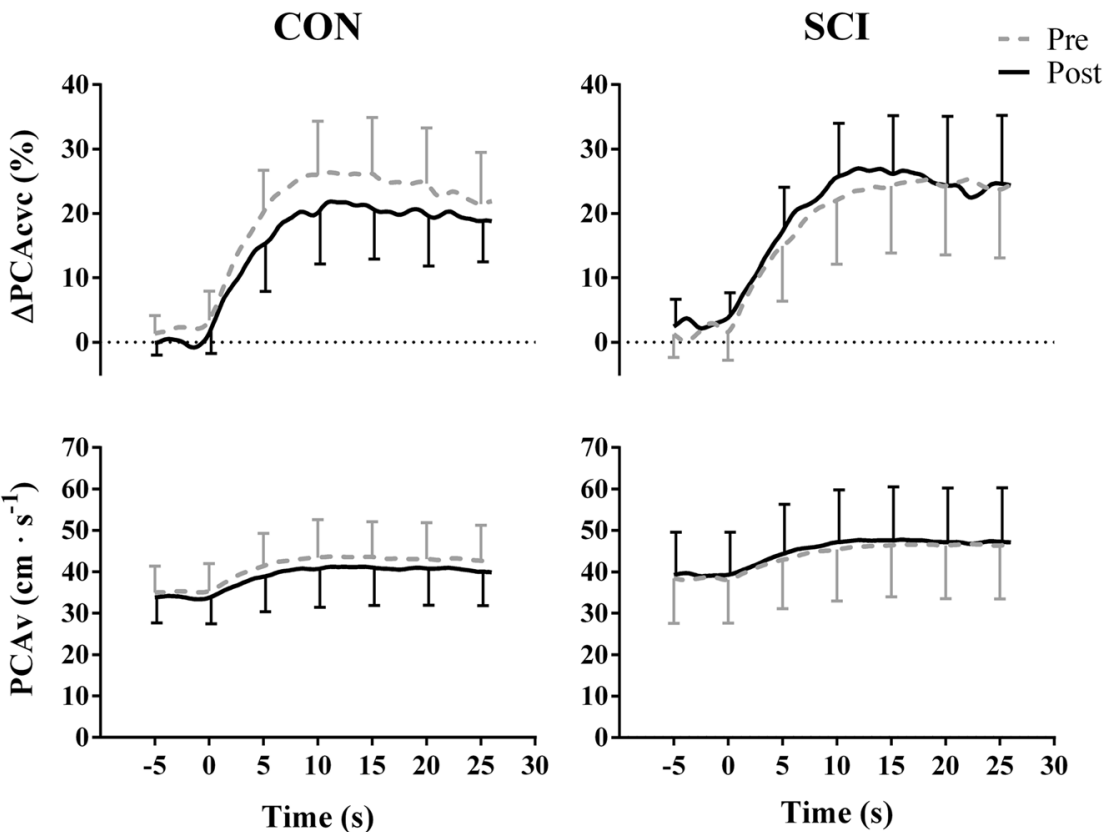

(8.8)\% in SCI and $\mathrm{CON}$, respectively, and remained unchanged after heating at 29.7 (8.6) vs. 25.2 (8.8)\% in SCI and $\mathrm{CON}$, respectively (Fig. 1). Hemoglobin and hematocrit did not change with heating $(P>0.05)$, but there tended to be a main effect of time on white blood cell and platelet counts after heating $(P<0.01$ and $P=0.08$, respectively; Table 1).

\section{Discussion}

This study examined the cerebrovascular responses following acute heat stress in individuals with chronic SCI. The primary outcomes of this study were that $\mathrm{CBF}$ and NVC were maintained during increases in Tcore in SCI. Although it is established that $\mathrm{CBF}$ declines with heat stress as a result of hyperthermia-induced hypocapnia and consequent cerebral vasoconstriction (reviewed in Bain et al [25].), we did not observe this response. This is likely because hyperventilation occurs during heat stress with a change in Tcore $>0.5^{\circ} \mathrm{C}$ [19] and the SCI group in this study was only moderately above this threshold and the $\mathrm{CON}$ group was below it. Moreover, $\mathrm{P}_{\mathrm{ET}} \mathrm{CO}_{2}$ decreased (main effect of time $P=0.06$ ) by only $2 \mathrm{mmHg}$ in both groups (Table 1), whereas slightly larger decreases might be required to reduce CBF [19]. These results suggest that individuals with SCI may have a lower ventilatory sensitivity (i.e., hyperventilatory response) to heat stress considering the similar decreases in $\mathrm{P}_{\mathrm{ET}} \mathrm{CO}_{2}$ with differing Tcore responses compared to $\mathrm{CON}$. Indeed, $\mathrm{P}_{\mathrm{ET}} \mathrm{CO}_{2}$ is inversely proportional to alveolar ventilation assuming a constant $\mathrm{VCO}_{2}$, which is the case $<1.0{ }^{\circ} \mathrm{C}$ increase in Tcore
[19]. Yet, these results nonetheless demonstrate the safety and potential application of lower limb heating as a therapy in SCI. Caution should be applied, however, when exposing individuals with SCI to greater degrees of heat stress given the blunted sweating and cutaneous vasodilation responses below the level of injury, particularly in high level (i.e., tetraplegic) injuries [26]. Individuals with SCI will store heat at a greater rate during external heating compared to CON (as observed in the current study) because of their smaller body mass, which also likely explains in large part their lower resting Tcore due to lower basal metabolic rate [27]. Nevertheless, the use of the lower limb heating protocol (compared to whole-body heating) permitted high tolerance in SCI participants to mild-moderate increases in Tcore, and no adverse events occurred including any bouts of autonomic dysreflexia.

In contrast to some other studies of acute heat stress in uninjured participants, we observed smaller changes in MAP and no effect of heating on carotid-femoral PWV, which might be explained by the relatively mild changes in Tcore in this study. For example, $30 \mathrm{~min}$ of hot water immersion (up to the sternum) [13] induced reductions in MAP of up to $20 \mathrm{mmHg}$ and reductions of PWV of $\sim 1.0 \mathrm{~m} / \mathrm{s}$ in uninjured populations. However, we observed increases in MAP similar to that reported by Shibasaki et al [28]. in participants with tetraplegia compared to both uninjured and individuals with paraplegia, although this response remains unexplained. It is noteworthy that in the study reporting reduced MAP and PWV, Tcore increased by $\sim 2.0^{\circ} \mathrm{C}$ [13]. Such extreme increases in Tcore highlight the acute benefits of passive heating on the cardiovascular system, but this magnitude of heating may not be necessary 
to induce chronic positive adaptations. Indeed, Bailey et al [16]. demonstrated that after eight weeks of repeated hot water immersion, with increases in Tcore $\sim 0.6^{\circ} \mathrm{C}, \mathrm{MCAv}$ was increased in uninjured young women. Moreover, it was recently reported that molecular responses to eight weeks of heat therapy (e.g., increased heat shock proteins) reduced inflammation and reactive oxygen species production during hypoxia-reoxygenation [29]. These data indicate potentially promising results for endothelial cell protection during ischemia-reperfusion in vivo and could be relevant for cerebral protection following strokes; yet, the translation to human cerebral endothelial function in vivo has yet to be determined. Together, the indirect benefits to cardiovascular risk factors and direct effects of heat on the brain (e.g., $\mathrm{Q}_{10}$ effect, regionally increased metabolism [30], etc.) make passive heating a promising intervention for cerebral vascular health in SCI.

Limitations: Compared to previous reports $[6,21]$, it is currently unclear why we did not observe lower NVC responses in the SCI group compared to $\mathrm{CON}$ at baseline. The supine position used throughout our protocol and similar blood pressures between groups [6] is likely the major factor since normalization of blood pressure in SCI while sitting has previously been shown to also normalize NVC [6]. The lower statistical power of the ANOVA used in this current study could also explain the difference in baseline NVC between this study and other cross sectional studies [21], as well as the absence of changes in primary outcomes of CBF and NVC before and after heating in SCI. When data were analyzed using a paired t-test instead, there were was no between-group difference in NVC $(P=0.42)$, nor were there differences in $\mathrm{CBF}$ or $\mathrm{NVC}$ following heating in SCI only ( $P=0.88$ and $P=0.71$, respectively). Moreover, the nationality of the participants in the current study is another difference compared to previous studies (i.e., Croatian vs. Canadian). Considering their lower body mass and probable impairments of sweating and skin blood flow, it is not surprising that core temperature increased to a greater extent in the SCI group in the current study. We also observed no differences in the CBF or NVC responses following heating in either group, but the vascular responses to heating between groups should be carefully interpreted in view of the significant interaction between group and time on core temperature.

Nevertheless, the purpose of this study was to determine the effects of a practical form of heating on cerebrovascular function in SCI. After an hour of lower limb heating, Tcore increased by $\sim 0.7^{\circ} \mathrm{C}$ in the SCI group indicating that this method of heating does not predispose participants to heat injury despite their thermoregulatory impairment. In uninjured individuals, other studies should aim to characterize NVC with greater increases in core temperature similar to that in the SCI group in the current study. The decreased
MCA conductance $(-10 \%)$ in SCI suggests that despite maintained $\mathrm{CBF}$, intracranial conductance could be impaired in SCI. Finally, the mechanisms contributing to a lower ventilator sensitivity to heat in SCI warrants elucidation. Future studies should consider quantifying cerebrovascular conductance at more severe increases in Tcore to determine a possible threshold at which heat stress might be counterproductive in SCI.

Conclusion: The results of the present study indicate that a feasible method (i.e., lower limb heating) of mild acute heating does not impair or improve cerebrovascular function in SCI. Further study of the effects of chronic heating interventions are needed to establish any potential benefits for cerebrovascular health in SCI.

\section{Data archiving}

The individual datasets generated and/or analysed during the current study are available from the corresponding author on reasonable request.

Acknowledgements We thank the participants for volunteering their time for this study.

Funding This study was supported in part by a Seed Grant from ICORD supported by the Blusson Integrated Cures Partnership (PNA), Canada Research Chairs program (PNA), and University of Calgary Start-up funds (AAP).

Author contributions GBC and PNA. conceived and designed the study, collected data, interpreted results, and drafted the manuscript. OB, AAP, TM, ZKS, AHXL, JWS, AVK, and ZD collected data and interpreted results. HGC analysed and interpreted data. All authors critically revised the manuscript and approved the final version. All authors agree to be accountable for all aspects of the work in ensuring that questions related to the accuracy or integrity of any part of the work are appropriately investigated and resolved. All persons designated as authors qualify for authorship, and all those who qualify for authorship are listed.

\section{Compliance with ethical standards}

Ethics We certify that all applicable institutional and governmental regulations concerning the ethical use of human volunteers were followed during the course of this research.

Conflict of interest The authors declare that they have no conflict of interest.

Publisher's note: Springer Nature remains neutral with regard to jurisdictional claims in published maps and institutional affiliations.

\section{References}

1. Cragg JJ, Noonan VK, Krassioukov A, Borisoff J. Cardiovascular disease and spinal cord injury. Neurology. 2013;81:723-8.

2. Phillips AA, Krassioukov AV, Ainslie PN, Warburton DER. Perturbed and spontaneous regional cerebral blood flow responses 
to changes in blood pressure after high-level spinal cord injury: the effect of midodrine. J Appl Physiol. 2014;116:645-53.

3. Phillips AA, Matin N, Jia M, Squair JW, Monga A, Zheng MMZ, et al. Transient hypertension after spinal cord injury leads to cerebrovascular endothelial dysfunction and fibrosis. J Neurotrauma. 2017;35:573-81.

4. Wolters FJ, Zonneveld HI, Hofman A, van der Lugt A, Koudstaal PJ, Vernooij MW, et al. Cerebral perfusion and the risk of dementia: a population-based study. Circulation. 2017;136: 719-28.

5. Pires PW, Dams Ramos CM, Matin N, Dorrance AM. The effects of hypertension on the cerebral circulation. Am J Physiol-Heart Circ Physiol. 2013;304:H1598-H1614.

6. Phillips AA, Warburton DE, Ainslie PN, Krassioukov AV. Regional neurovascular coupling and cognitive performance in those with low blood pressure secondary to high-level spinal cord injury: improved by alpha-1 agonist midodrine hydrochloride. J Cereb Blood Flow Metab. 2014;34:794-801.

7. Phillips AA, Chan FH, Zheng MMZ, Krassioukov AV, Ainslie PN. Neurovascular coupling in humans: Physiology, methodological advances and clinical implications. J Cereb Blood Flow Metab. 2016;36:647-64.

8. Wecht JM, Weir JP, Katzelnick CG, Wylie G, Eraifej M, Nguyen $\mathrm{N}$, et al. Systemic and cerebral hemodynamic contribution to cognitive performance in spinal cord injury. J Neurotrauma. 2018;35:2957-64.

9. Phillips AA, Matin N, Frias B, Zheng MMZ, Jia M, West C, et al. Rigid and remodelled: cerebrovascular structure and function after experimental high-thoracic spinal cord transection. J Physiol. 2016;594:1677-88.

10. van den Berg-Emons RJ, Bussmann JB, Haisma JA, Sluis TA, van der Woude LH, Bergen MP, et al. A prospective study on physical activity levels after spinal cord injury during inpatient rehabilitation and the year after discharge. Arch Phys Med Rehabil. 2008;89:2094-101.

11. Romero SA, Gagnon D, Adams AN, Cramer MN, Kouda K, Crandall CG. Acute limb heating improves macro and microvascular dilator function in the leg of aged humans. Am J PhysiolHeart Circ Physiol. 2016;312:H89-H97.

12. Brunt VE, Howard MJ, Francisco MA, Ely BR, Minson CT. Passive heat therapy improves endothelial function, arterial stiffness and blood pressure in sedentary humans. J Physiol. 2016;594:5329-42.

13. Thomas KN, van Rij AM, Lucas SJE, Cotter JD. Lower-limb hotwater immersion acutely induces beneficial hemodynamic and cardiovascular responses in peripheral arterial disease and healthy, elderly controls. Am J Physiol-Regul Integr Comp Physiol. 2016;312:R281-R291.

14. Hoekstra SP, Bishop NC, Faulkner SH, Bailey SJ, Leicht CA. Acute and chronic effects of hot water immersion on inflammation and metabolism in sedentary, overweight adults. J Appl Physiol. 2018;125:2008-18.

15. Hashizaki T, Nishimura Y, Teramura K, Umemoto Y, Shibasaki M, Leicht CA, et al. Differences in serum IL-6 response after $1{ }^{\circ} \mathrm{C}$ rise in core body temperature in individuals with spinal cord injury and cervical spinal cord injury during local heat stress. Int $\mathbf{J}$ Hyperth. 2018;35:541-7.
16. Bailey TG, Cable NT, Miller GD, Sprung VS, Low DA, Jones H. Repeated warm water immersion induces similar cerebrovascular adaptations to 8 weeks of moderate-intensity exercise training in females. Int J Sports Med. 2016;37:757-65.

17. Laukkanen $T$, Kunutsor S, Kauhanen J, Laukkanen JA. Sauna bathing is inversely associated with dementia and Alzheimer's disease in middle-aged Finnish men. Age Ageing. 2017;46: 245-9.

18. Kunutsor SK, Khan H, Zaccardi F, Laukkanen T, Willeit P, Laukkanen JA. Sauna bathing reduces the risk of stroke in Finnish men and women: a prospective cohort study. Neurology. 2018;90: e1937-e1944.

19. Fan J-L, Cotter JD, Lucas RAI, Thomas K, Wilson L, Ainslie PN. Human cardiorespiratory and cerebrovascular function during severe passive hyperthermia: effects of mild hypohydration. J Appl Physiol. 2008;105:433-45.

20. Coombs GB, Barak OF, Phillips AA, Mijacika T, Sarafis ZK, Lee AHX, et al. Acute heat stress reduces biomarkers of endothelial activation but not macro- or microvascular dysfunction in cervical spinal cord injury. Am J Physiol-Heart Circ Physiol. 2018;316: H722-H733.

21. Squair JW, Lee AH, Sarafis ZK, Chan F, Barak OF, Dujic Z et al. Network analysis identifies consensus physiological measures of neurovascular coupling in humans. J Cereb Blood Flow Metab. 2019. https://doi.org/10.1177/0271678X19831825.

22. Woodman RJ, Playford DA, Watts GF, Cheetham C, Reed C, Taylor RR, et al. Improved analysis of brachial artery ultrasound using a novel edge-detection software system. J Appl Physiol. 2001;91:929-37.

23. Laurent S, Cockcroft J, Van Bortel L, Boutouyrie P, Giannattasio C, Hayoz D, et al. Expert consensus document on arterial stiffness: methodological issues and clinical applications. Eur Heart J. 2006;27:2588-605.

24. Miyatani M, Alavinia SM, Szeto M, Moore C, Craven BC. Association between abnormal arterial stiffness and cardiovascular risk factors in people with chronic spinal cord injury. Eur J Prev Cardiol. 2017;24:552-8.

25. Bain AR, Nybo L, Ainslie PN. Cerebral vascular control and metabolism in heat stress. Compr Physiol. 2015;5:1345-80.

26. Price MJ. Thermoregulation during exercise in individuals with spinal cord injuries. Sports Med. 2006;36:863-79.

27. Mollinger LA, Spurr GB, el Ghatit AZ, Barboriak JJ, Rooney CB, Davidoff DD, et al. Daily energy expenditure and basal metabolic rates of patients with spinal cord injury. Arch Phys Med Rehabil. 1985;66:420-6.

28. Shibasaki M, Umemoto Y, Kinoshita T, Kouda K, Ito T, Nakamura $\mathrm{T}$, et al. The role of cardiac sympathetic innervation and skin thermoreceptors on cardiac responses during heat stress. Am J Physiol Heart Circ Physiol. 2015;308:H1336-H1342.

29. Brunt VE, Wiedenfeld-Needham K, Comrada LN, Minson CT. Passive heat therapy protects against endothelial cell hypoxiareoxygenation via effects of elevations in temperature and circulating factors. J Physiol. 2018;596:4831-45.

30. Nunneley SA, Martin CC, Slauson JW, Hearon CM, Nickerson LDH, Mason PA. Changes in regional cerebral metabolism during systemic hyperthermia in humans. J Appl Physiol. 2002;92:846-51. 